\title{
Patient distress after radiotherapy to the head and neck was often hidden
}

\author{
Wells $M$. The hidden experience of radiotherapy to the head and neck: a qualitative study of patients after completion of \\ treatment.J Adv Nurs 1998 Oct;28:840-8.
}

\section{Question}

What are the experiences of patients with head and neck cancer during the month after completion of radiotherapy?

\section{Design}

Naturalistic inquiry.

\section{Setting}

Oxford, UK.

\section{Patients}

12 patients who received radical radiotherapy to squamous cell cancers in the head and neck area during a 4 month period.

\section{Methods}

After patients completed a course of radiotherapy, they were given diaries to record important events and feelings. Approximately 1 month after the completion of radiotherapy, indepth interviews were held in patients' homes. At the end of the interviews, patients were presented with a series of symptom cards. These were intended to prompt patient discussion of symptoms that might not have been addressed and provide opportunities for discussion of sensitive topics (eg, problems with kissing).

\section{Main findings}

The distress experienced by patients during the month after radiotherapy was often hidden. Patients downplayed their experience, and many showed resignation or acceptance of the cancer (eg, "that's how life goes"). They tried to convince themselves that things were really all right, often comparing themselves favourably with other cancer patients who were "worse off."

Unpleasant symptoms induced by treatment disrupted patients' day to day lives. Physical side effects included tiredness, sore and dry mouth or throat, and difficulty swallowing and sleeping. Sev- eral patients developed severe skin and mucosal reactions, which further exacerbated problems with eating, breathing, and sleeping.

Patients felt that treatment induced problems were insignificant in comparison with their cancer, and they did not want to take up professionals' valuable time with these "insignificant" concerns. The diaries revealed the considerable distress that patients experienced with daily ups and downs and in maintaining the delicate balance between feeling physically and emotionally well and feeling overwhelmed by symptoms and fears.

Physical symptoms from radiotherapy were most severe during the first few weeks after radiotherapy. Emotionally, however, patients continued to experience a loss of self (self confidence and self worth) and they felt that their lives were "on hold." Improvement in physical symptoms and resumption of normal activities helped patients to believe they were recovering.

Living with uncertainty was a prime source of distress. The period after completion of radiotherapy was often the first opportunity some patients had to think about what was happening to them, but they had no one to talk with about their feelings. They felt that clinicians were not easily accessible once treatment was finished. Patients attached importance to visits to their doctors, and wished their doctors had shown more interest in them after completion of radiotherapy when they wanted to share their experiences and find out what to expect.

\section{Conclusions}

The physical and emotional distress experienced by patients in the month after completing radiotherapy for head or neck cancer was tacitly accepted by patients and professionals as a normal and insignificant result of the treatment. Patient distress therefore remained a hidden experience.

Source of funding: no external funding

For correspondence: Mary Wells, Department of Radiotherapy and Oncology, Ninewells Hospital, Dundee DD1 9SY,UK. Fax (0)1382 496232.

\section{Commentary}

Limited research exists on the impact of radiotherapy on patients with head and neck cancer. The study by Wells therefore makes an important contribution to our understanding of how these patients experience radiotherapy and uncovers some of the hidden aspects of recovery after radiotherapy. The main findings indicate that patients consistently underreported radiotherapy symptoms, played down the impact the treatment had on their lives, and had little support or follow up after the course of treatment was completed. These findings highlight the importance of providing emotional support during and after treatment, as well as the need for strategic follow up of these patients. Follow up is especially important because many side effects of radiotherapy occur 1 month after treatment, which, as
Wells rightly points out, is when the support of specialist nurses and radiotherapy staff is often reduced as patients no longer attend the cancer centre for daily treatment.

Nurses involved in the care and support of this client group need to consider how they can best assist patients to cope with the side effects of radiotherapy, legitimise patients' experiences, and help them to deal with uncertainty. Nurses working in this clinical area could support patients by providing individualised written information, drop-in clinics, telephone support, self help groups, and effective liaison with community support staff.

This study's naturalistic design offers a rich description of the experience of a specific group of patients undergoing radiotherapy and suggests some useful ways in which care for these patients could be improved, such as providing a rehabilitative approach to follow up, access to specialist head and neck cancer nurses, and liaison with primary care. The implications for practice could have been strengthened if the author had discussed the results in the context of high quality evaluative research (ie, randomised controlled trials of interventions that are effective in reducing patient distress). None the less, the study findings add to nurses' understanding of the experiences of patients with cancer of the head and neck and highlight areas where nursing interventions may be needed.

$$
\begin{array}{r}
\text { Karen Cox, RGN, BSc(Hons) } \\
\text { Lecturer, School of Nursing } \\
\text { University of Nottingham } \\
\text { Nottingham, UK }
\end{array}
$$

